\title{
I. NICOLAS FOUQUET: QUO NON ASCENDET?
}

\section{Die frühen Jahre (1615-1630)}

Nicolas Fouquet wurde 1615 als zweitältester Sohn Marie de Maupeous (1590-1681) und François Fouquets (1587-1640) in Paris geboren. Die Fouquets $^{1}$ stammen aus der Bretagne und führen in ihrem Wappen ein halb aufgerichtetes und mit den Vorderpfoten nach oben weisendes Eichhörnchen, das in der Mundart ihrer Heimat »fouquet « genannt wird. Als François Fouquet im September 1627 das Amt eines conseiller d'État en service ordinaire übernahm, fügte er seinem Wappen die Devise »Quo non ascendet? « hinzu und gab damit der Hoffnung auf einen sozialen und politischen Aufstieg seiner Familie Ausdruck ${ }^{2}$. Tatsächlich sah die Zukunft der Fouquets vielversprechend aus: Noch im 15. Jahrhundert als Tuchhändler in Angers ansässig, hatten sie sich im 16. Jahrhundert erfolgreich um ihren gesellschaftlichen Aufstieg bemüht. Durch den Kauf von Ländereien, eheliche Verbindungen mit dem lokalen Patriziat und eine umfassende Ausbildung der Familienmitglieder konnte 1578 mit dem Großvater Nicolas' der erste Fouquet als conseiller au parlement de Paris in königliche Dienste treten. Sein Sohn verkaufte das Amt 1615 für das eines maître des requêtes und wurde damit sowohl Mitglied des Parlaments als auch des Conseil d'État, des königlichen Rates ${ }^{3}$. Nach der Ernennung des Kardinals Richelieu zum Premierminister im Jahre 1624 wurde er zu dessen privatem Geldgeber und Vertrauten. Richelieu weckte das Interesse François Fouquets für die Seefahrt und den überseeischen Handel, die von nun an zu den Gebieten gehörten, auf denen sich die Familie engagierte $^{4}$. In nur drei Generationen waren die Fouquets damit von Kaufleuten

${ }^{1}$ Der Name schrieb sich ursprünglich nach alter Mode mit einem »c« (Foucquet).

2 Die von dem Abbé de Choisy geprägte Form der Devise »Quo non ascendam? « ist falsch; man findet sie trotzdem des Öfteren in der Literatur über Fouquet, zum Beispiel bei Mongrédien, Foucquet.

${ }^{3}$ Es ist unwahrscheinlich, dass mit der Übernahme dieses Amtes 1615 bereits eine Nobilitierung der Familie verbunden war. Die maîtres des requêtes gehörten der robe, der Klasse der Justiz-, Polizei- und Verwaltungsbeamten an, die sowohl aus der haute bourgeoisie als auch aus dem Adel stammten: »Le mot >robe< [...] réunit, dans une même profession, ces gens de toute origine, de tout niveau de fortune, et de rang social variable [...]. La robe n'est point une classe sociale [...], mais [...] >la classe qui lit« (BLuche, »Robe«, in: Dictionnaire du Grand Siècle [DGS], S.1344). Die bourgeoisie de robe, ein in diesem Kontext von Petitfils verwendeter Begriff (Fouquet, S.258), war aufgrund der damit verbundenen Privilegien und gesellschaftlichen Stellung daran interessiert, in die noblesse de robe aufzusteigen, doch erst 1644 erhielten sie das Privileg zum anoblissement au premier degré, einer Nobilitierung nach zwanzigjähriger Amtszeit.

${ }^{4}$ François Fouquet besaß Anteile an zahlreichen Handelsgesellschaften, wie der Compagnie des îles d'Amérique, der Compagnie de Nouvelle France und der unter anderem von ihm gegründeten Compagnie des Indes orientales (vgl. GreLL, Introduction, S.9). 
in Angers zu engen Mitarbeitern der politischen Elite Frankreichs aufgestiegen.

Das Streben nach gesellschaftlichem Aufstieg paarte sich in der Familie Fouquet mit einem Hang zu Kultur und Gelehrsamkeit. Die Förderung von Kunst und Literatur bot der aufsteigenden bourgeoisie de robe die Möglichkeit zur sozialen Distinktion. Herrschaftliche Bauten, Aufsehen erregende Kunstsammlungen, literarische Lobreden und festliche Empfänge waren geeignete Mittel, Ansehen zu erwerben und die eigene Stellung in Gesellschaft und Politik zu festigen ${ }^{5}$. Besonders auf literarischem Gebiet sah die erste Hälfte des 17. Jahrhunderts zahlreiche Neuerungen: Das Hôtel Rambouillet schuf mit seiner Chambre bleue seit 1613 eine Salonkultur, die das kulturelle Leben der Stadt für lange Zeit prägen sollte. Sie wurde zu einem Gegenpol der von Richelieu 1635 gegründeten Académie française, welche fortan versuchte, das literarische Schaffen politisch zu lenken ${ }^{6}$.

Die Fouquets gehörten zu dem Kreis der Parlamentsräte, die im Herzen von Paris Künstler und Wissenschaftler empfingen und die Salonkultur pflegten. In die Rue de Jouy, mitten in dem von Gärten und dekorativen Fassaden geprägten Marais, luden sie Gäste von Rang und Namen, unter ihnen den Lieblingsdichter Richelieus, Boisrobert, der seinem Wohlwollen der Familie Fouquet gegenüber in einer Epistel an Basile Fouquet mit folgenden Worten Ausdruck verlieh:

Ton père aux heures familières

Avoit des preuves singulières

De son adorable bonté

Dont l'univers fut enchanté?.

Nicolas' Vater François Fouquet gehörte zu jenen, die sich mit Leidenschaft wissenschaftlichen Studien hingaben und ein breites Interesse an künstlerischen und literarischen, besonders jedoch an wissenschaftlichen Neuerungen zeigten. Die Gebiete seiner Sammel- und Fördertätigkeit waren nicht ohne Bedacht gewählt. Das kulturelle Engagement ergänzte seine politischen Aktivitäten und verfolgte das Ziel gesellschaftlicher Anerkennung. Die 15000 Bände umfassende Bibliothek François Fouquets war eine der größten privaten Bibliotheken in ganz Paris. Selbst die Sammlung des Premierministers blieb mit 6380 Bänden hinter derjenigen seines engen Mitarbeiters zurück. François Fouquet schuf auf diese Weise einen der Forschung förderlichen Raum der Gelehrtheit ${ }^{8}$. Einen Schwerpunkt seiner Sammlung bildete die Geogra-

5 Zum nonroyalen Mäzenatentum vor 1661 siehe: Mousnier, Mesnard, Âge d'or.

6 Vgl. Fumaroli, La Fontaine, S.164. Am 30. Mai 1631 erschien außerdem die erste Ausgabe der »Gazette de France«, die fortan das Zeitgeschehen dokumentierte.

7 Boisrobert, Épistres, Buch 3, Epistel V, S. 129.

${ }^{8}$ Es ist allerdings nicht bekannt, ob François Fouquet seine Bibliothek der gelehrten Öffentlichkeit zugänglich machte. Wahrscheinlich ist aber, dass zumindest den Wissenschaftlern und Literaten aus seinem Kreis die Nutzung des großen Buchbestandes erlaubt war. 
phie mit einer großen Kartensammlung ${ }^{9}$, die - so ist anzunehmen - nicht nur dem Interesse an den Entdeckungen des vergangenen Jahrhunderts geschuldet war, sondern vor allem mit der Beteiligung François Fouquets an den in Übersee tätigen Handelsgesellschaften zu erklären ist.

Fouquets Mutter Marie de Maupeou hatte sich auf die Herstellung von Medikamenten >de bonne femme< spezialisiert. Ihre Studien waren erfolgreich: Im Jahre 1675 gab sie in Zusammenarbeit mit Delescure, einem Gelehrten der Universität von Montpellier, den »Recueil de recettes choisies expérimentées et approuvées contre quantité de maux fort communs, tant internes qu'externes, invétérés ou difficiles à guérir « heraus, der nach mehreren Auflagen im Jahre 1687 in einer Folgeausgabe vervollständigt wurde. Dem königlichen Hause waren ihre Kenntnisse von lebensrettender Hilfe: Als im Jahre 1664 nach einer Frühgeburt die Königin dem Tode nahe schien und der Hof bereits alle Hoffnung auf ihre Rettung aufgegeben hatte, führte ein Heiltrunk Mme Fouquets die Genesung Maria Theresias herbei ${ }^{10}$. Ihre Passion für die Heilkräuterkunde gab sie an ihren Sohn weiter, den sie in der Herstellung von Medikamenten und der Diagnose von Krankheitssymptomen unterrichtete ${ }^{11}$.

Nicolas Fouquet wurde durch die in seinem Elternhaus gepflegte Lebensart stark geprägt und kam hier in nähere Berührung mit Literatur und Naturwissenschaft ${ }^{12}$. Seine humanistische Ausbildung begann er im Alter von zwölf Jahren auf dem Jesuitenkolleg von Clermont, an dessen Wiedereröffnung der Vater im Jahre 1618 maßgeblich beteiligt war. Hier traf er auf Mitschüler aus angesehenen Familien: Söhne von Prinzen, Staatssekretären, Botschaftern, Räten und Präsidenten, aber auch Sprösslinge reicher Kaufleute und Händler ${ }^{13}$. Die in dieser Zeit geknüpften Bekanntschaften sollten ihm ein Leben lang von Nutzen sein und halfen ihm später beim Aufbau eines Netzwerkes von Beziehungen ${ }^{14}$. Die Ausbildung Nicolas' entsprach dem klassischen Ka-

9 CoRdey, Bibliothèque, S. 6: »Son père, le conseiller au Parlement François Fouquet, possédait une importante collection de cartes géographiques, les deux plus beaux globes connus, l'un terrestre, l'autre céleste, et une bibliothèque de livres soigneusement choisis «.

10 SÉvigné, Lettres, Bd.I, S.123, Nr.33, Brief der Marquise an M. de Pomponne vom 20. November 1664: »Mme Foucquet la mère a donné un emplâtre à la Reine, qui l'a guérie de ses convulsions, qui étoient à proprement parler des vapeurs«.

11 Während seiner Gefangenschaft in Pignerol ließ sich Fouquet ein kleines Chemielabor zusammenstellen und vertrieb sich die Zeit mit pharmazeutischen Forschungen und der Herstellung von Medikamenten, die seine körperlichen Leiden zu lindern halfen.

${ }^{12}$ François Fouquet erkannte das breite Interesse für Kunst und Kultur seines zweitältesten Sohnes und erwählte ihn zum Erben seiner Antiquitäten und Bücher: »Je lui donne mes livres et antiquités, qui ne seroient considérables, s'ils étoient partagés « (aus dem Testament François Fouquets, zitiert nach: Chatelain, Foucquet, S.12).

13 Jean-Baptiste Poquelin, Sohn eines Dekorateurs, folgte sechs Jahre später auf den gleichen Schulbänken den Lehren der Jesuiten.

14 Der soziologische Begriff Netzwerk wurde in den späten siebziger Jahren unter dem Namen Verflechtung von Wolfgang ReINHARD in die Geschichtswissenschaft eingeführt 
non: Er lernte Griechisch und Latein, das auch als Unterrichtssprache diente. Ziel der collèges der Jesuiten war es nicht, Gelehrte heranzubilden, sondern das Auffassungsvermögen ihrer Zöglinge zu schulen und sie zu honnêtes hommes chrétiens heranzuziehen, die als humanistisch gebildete Christen fähig waren, sich in der Welt zurechtzufinden und Verantwortung im Staat zu übernehmen. Die Verfeinerung der Urteilsfähigkeit und des Geschmacks sowie die Förderung vielseitiger Interessen und der Selbstbeherrschung sollten aus den Zöglingen universal gebildete Persönlichkeiten machen ${ }^{15}$. Das Curriculum der Jesuitenkollegs legte einen Schwerpunkt auf die sprachliche Ausbildung der Eleven: »Ils enseignent les langues, les préceptes de la dialectique et expliquent à leurs élèves dans la mesure du possible la rhétorique [et] [...] cultivent les belles-lettres «16. Hinzu trat die Belehrung in Naturwissenschaft, Geschichte und Geographie. Ein wichtiger Bestandteil war zudem die éducation par le jeu, die einherging mit der Liebe zu Festen: »La diffusion élargie d'un répertoire où se côtoient la mythologie, les allégories, les emblèmes, les fictions chevaleresques est l'un des facteurs intellectuels les plus marquants du développement de l'effimero barocco ${ }^{17}$.

Fouquet, der das Collège de Clermont von seinem zwölften bis sechzehnten Lebensjahr besuchte, blieb den Jesuiten sein Leben lang verbunden und wurde zu einem beständigen Förderer ihrer Einrichtungen ${ }^{18}$. Von ihren Erziehungsmethoden wurde er nachhaltig geprägt. Sie festigten seinen Geschmack an Literatur und Geschichte, der in der Förderung von Literaten und dem Aufbau einer großen Bibliothek seinen Ausdruck fand. Sie weckten zudem seine Vorliebe für aufwendige Theateraufführungen und festliche Inszenierungen, die am 17. August 1661 ihren Höhepunkt finden sollte.

\section{Die ersten Berufsjahre (1631-1652)}

Nicolas Fouquet profitierte von den ehrgeizigen Bestrebungen seines Vaters, der durch die Förderung seiner Kinder mit allen Kräften auf den weiteren gesellschaftlichen Aufstieg der Familie hinarbeitete. Nach dem Ende seiner schulischen Ausbildung wurde Nicolas im Januar 1631 Schatzmeister der Abtei Saint-Martin in Tours und löste seinen Großonkel Isaac in diesem

und meint den Zusammenschluss von Personen zu einem Gefüge von Beziehungen, bei dem jeder die entstehenden Kontakte für seine Zwecke einzusetzen versucht. Im 17. Jahrhundert war es üblich, dass ein Staatsmann sein persönliches Netzwerk aufbaute, indem er Klienten in einem Treueverhältnis an sich band (siehe S.18).

15 Vgl. Chanut, »Jésuites«, in: DGS, S. 790.

16 Aus: Sturm, Jean, Classicae Epistolae, sive Scholae Argentiniensis restituae, o. O. 1565; zitiert nach: Chartier, Compère, Julia, Éducation, S. 159.

17 Choué, »Fêtes «, in: DGS, S. 586. Zum Curriculum siehe auch: Dainville, Éducation.

18 Zur Finanzierung der Bibliothek des Collège Clermont durch den Finanzminister: siehe Kapitel 2.2. 
Amt ab. Mit achtzehn Jahren erwarb er an der Sorbonne die licence ès lois und schrieb sich als Rechtsanwalt ein. Bereits kurz darauf, im Jahre 1633, kaufte ihm sein Vater das Amt eines conseiller in dem von Richelieu gegründeten Parlament von Metz ${ }^{19}$. Im folgenden Jahr ging Nicolas als Beobachter in das von der französischen Armee besetzte Lothringen und trat 1636, dank der finanziellen Unterstützung seines Vaters und der Fürsprache Richelieus $^{20}$, mit gerade einundzwanzig Jahren das Amt eines maître des requêtes de l'hôtel de Paris an. Die Vermählung mit Louise Fourché (16201641) am 24. Januar 1640, die dem jungen Fouquet eine Mitgift von 160000 Livres sowie die »terre noble de Quéhillac en Bourron« einbrachte, gab ihm eine vielversprechende finanzielle Basis für seine weitere Karriere. Die Heirat ermöglichte ihm den Kauf der Domäne Vaux-le-Vicomte, die eine sichere Geldanlage darstellte und ihm zugleich den Titel eines vicomte verlieh ${ }^{21}$. Rund zweihundert Kaufverträge der folgenden Jahre belegen den Wunsch Fouquets, seinen Besitz zu vergrößern und eine »propriété de prestige ${ }^{22} \mathrm{zu}$ schaffen - ein Sprungbrett, um in die noblesse de robe, den Amtsadel, aufzusteigen und sich im Landadel zu etablieren ${ }^{23}$. Obwohl bereits in den 1640er Jahren Abrissarbeiten am alten Schloss von Vaux sowie erste Arbeiten im Garten erfolgten, hatte Fouquet zunächst nicht die Absicht, die Domäne zu einem neuen Wohnsitz auszubauen. Den Papieren Olivier d'Ormessons zufolge wurde zunächst lediglich ein Nutzgarten angelegt und ein Wirtschaftshof errichtet ${ }^{24}$.

19 Bereits im November 1631 hatte François Fouquet bei Richelieu um das Amt eines conseiller im Parlament der Bretagne für seinen Sohn gebeten (Brief François Fouquets an Richelieu vom 2. November 1631, in: Richelieu, Papiers, Bd. VI, S.644). In Metz erhielt Fouquet den Auftrag, zusammen mit Michel Marescot ein Inventar der Papiere der Stadt Vic zu erstellen, wo sich die chartes du temporel, die weltlichen Urkunden des Bistums von Metz, befanden.

${ }^{20}$ Heinrich IV. hatte das Mindestalter für die maîtres des requêtes auf 32 Jahre festgelegt, doch dank der Intervention Richelieus konnte Nicolas das Amt bereits im Alter von 21 Jahren antreten.

${ }^{21}$ Sowohl PetitfiLs, Fouquet, S.258, der von einer bourgeoisie de robe spricht, als auch Chatelain, Foucquet, S.9, zählen die Familie zur »haute bourgeoisie parlementaire qui confinait à la noblesse«. Obwohl es Anfang des 17. Jahrhunderts immer wieder zur Nobilitierung der bürgerlichen Parlamentsräte kam, muss man davon ausgehen, dass die Familie Fouquet erst nach 1644 in den zweiten Stand aufstieg.

22 Dessert, Fouquet, S. 150.

23 Siehe unter anderem AN MC ET/LI/ 511, 537, 538.

24 AN, Fonds d'Ormesson, 156 MI 18, fol. 79. Fouquets Arbeiten am Garten von Vaux fanden 1652 eine öffentliche Würdigung. Charles de Sercy widmete in diesem Jahr das von ihm herausgegebene Buch Claude MolLETS, »Theatre des plans et iardinages«, Nicolas Fouquet. In seiner Widmung lobt er die wunderbaren Gärten von Vaux-le-Vicomte, denen Tag für Tag neue Schönheiten hinzugefügt würden: »Ouy, Monseigneur, l'Agriculture a pour vous tant de charmes \& tant de délices, que vous ne dédaignez pas d'y occuper vos mains, \& d'y donner les plus précieux moments de vostre loisir; Vous cherchez dans l'innocence de la vie champestre des douceurs qu'on ne sçauroit rencontrer autre part: \& vous prenez plaisir de sortir des Parterres de Themis, pour entrer dans ces superbes Jar- 
Der Tod des Vaters im April 1640, dem eine Reihe von Todesfällen im Umkreis Fouquets folgten, machte Nicolas zum Oberhaupt der Familie sowie zum Verwalter des beträchtlichen väterlichen Vermögens ${ }^{25}$. Auch im Rat der westindischen Handelsgesellschaft nahm er den Platz des Vaters ein und gewann das Vertrauen Richelieus, der von nun an seine überseeischen Aktivitäten förderte. Im Februar 1641 starb der Großvater Gilles de Maupeou und hinterließ Nicolas die Verantwortung für die Familie seiner Mutter; im August desselben Jahres verschied seine Frau Louise, sechs Monate nach der Geburt der Tochter Marie. Das Jahr 1642 brachte dem jungen Fouquet erstmalig auch auf politischer Ebene einen immensen Verlust: Im Dezember starb Richelieu, der politische Förderer der Familie und seiner selbst. Für Nicolas, der nach dem Tod des Vaters zum engen Mitarbeiter des Kardinals aufgestiegen war, hätte dies eine Verzögerung, wenn nicht gar das Ende seines weiteren Aufstiegs bedeuten können, da mit dem Tod Richelieus dessen Verbindungen in die Regierungselite nicht mehr zugänglich waren ${ }^{26}$. Es zeigte sich jedoch, dass Fouquet in der königlichen Administration bereits so fest Fuß gefasst hatte, dass seiner weiteren Karriere selbst nach dem Tod Ludwigs XIII., der seinen Premierminister nur um einige Monate überlebte, und trotz der folgenden politischen Unruhen nichts im Wege stand. In Anna von Österreich, die im Namen ihres minderjährigen Sohnes Ludwig XIV. die Regentschaft übernahm, fand Fouquet eine neue mächtige Patronin, und es sollte sich zeigen, dass die Königstreue der Familie Fouquet gepaart mit dem politischen Geschick Nicolas' für seinen Aufstieg überaus förderlich war.

Seine diplomatischen Fähigkeiten konnte Fouquet erstmals im Jahre 1644 unter Beweis stellen. Ende 1643 wurde er durch eine persönliche Entscheidung der Königin zum intendant de Justice, Police et Finance in Grenoble ernannt. Obwohl ein Intendant kein direkter Vertreter des Königs in der Provinz war, vergrößerte sich sein Einflussbereich beständig und er gewann als Amtsgehilfe des Gouverneurs, der den König vertrat, zunehmend Macht und Ansehen.

Die wirtschaftliche Lage in den Provinzen war katastrophal: Der Krieg gegen Spanien erforderte hohe Summen, die in Form gnadenloser Besteuerung

dins de Vaux le Vicomte, où vous faites agreablement combattre l'Art avec la Nature, \& où vous adjoustez tous les iours de nouvelles beautez \& de nouveaux enrichissemens « (Mollet, Theatre des plans, S.III). Schriftliche oder bildliche Quellen, die den genauen Beginn der Gartenarbeiten belegen, existieren nicht.

25 Nicolas Fouquet war der zweite von sechs Söhnen von insgesamt zwölf Kindern. François V., der älteste Sohn und Erzbischof von Bayonne, wachte über die Einheit des Hauses sowie dessen moralische Integrität. Für die weltlichen Belange der Familie war Nicolas zuständig: »Ayant beaucoup avantage mon fils Nicolas, maître des requêtes par son contrat de mariage, je me promets qu'il aura soin particulier d'assister sa mère en la conduite de ses affaires et qu'il servira de père à ses frères et sœurs en la conduite de leur personne et de leur bien « (AN MC/ET/LI 505, 18. Avril 1640, provisions de François Fouquet).

26 Vgl. Dessert, Fouquet, S. 62. 
aus der Bevölkerung herausgepresst wurden ${ }^{27}$. Als Intendant war Fouquet für die Kontrolle des Einzugs der direkten Steuern zuständig. Bereits kurz nach seiner Ankunft in Grenoble zu Beginn des Jahres 1644 gewann er einen Einblick in die Situation und bat in einer Depesche an den Kanzler Séguier um einen königlichen Erlass, der der Bevölkerung eine Steuererleichterung im kommenden Jahr in Aussicht stellte. Fouquet hoffte, mit diesem Schritt die gespannte Stimmung, die sich in ersten, noch schwachen Protesten zeigte, bessern zu können ${ }^{28}$. Damit ergriff er öffentlich die Partei der Revoltierenden und stellte sich gegen die Regierung. Während er auf die Antwort aus Paris wartete, verließ Fouquet in Abwesenheit des Gouverneurs und ohne die Zustimmung des Kanzlers seinen Posten in Grenoble, um an der Inthronisierungszeremonie seines älteren Bruders François in Adge teilzunehmen. Als während seiner Abwesenheit die befürchteten Aufstände ausbrachen, forderte Séguier von der Königin mit Erfolg die Absetzung des Intendanten, der sich mit seinem ersten politischen Misserfolg konfrontiert sah. Fouquet trat den Rückweg nach Paris an. Während der Reise erreichten ihn zwei Briefe, die den Ausbruch weiterer Revolten meldeten. Da sein Nachfolger noch nicht eingetroffen, der Gouverneur des Dauphiné weit vom Unruheherd entfernt war und die Aufstandsbewegung auf weitere Teile des Landes überzugreifen drohte, beschloss Fouquet, sich persönlich um eine Entschärfung der Lage zu bemühen und reiste nach Valence, den Herd der Unruhen. Dort gelang es ihm mit viel Verhandlungsgeschick, Standhaftigkeit und Überzeugungskraft und trotz einer lebensbedrohlichen Szene, bei der seine Kutsche von rund fünfhundert Aufständischen überfallen wurde, die Revoltierenden zu beruhigen und die Gefahr eines weitflächigen Aufstandes abzuwenden.

Dieser Zwischenfall, bei dem Fouquet seine diplomatischen Fähigkeiten unter Beweis stellte, mag ihm erneut die Gunst der Regentin eingebracht und ein plötzliches Ende seiner Karriere abgewendet haben. Bereits im Frühjahr 1646 nahm Fouquet seine politische Tätigkeit wieder auf. Mit der Ernennung zum intendant à l'armée de Picardie im Mai 1647 und der Beförderung zum intendant de la généralité de Paris im folgenden Jahr hatte sich seine Stellung erneut gefestigt. Zugleich hatte Nicolas den 1643 von Anna von Österreich zum neuen Premierminister ernannten Jules Mazarin auf sein politisches Geschick aufmerksam gemacht. Die Bindung an Mazarin sollte für Fouquets weitere Karriere wegweisend sein.

Die schlechte finanzielle Situation des Landes sowie die Vermehrung und damit verbundene Wertminderung der Ämter riefen Anfang 1648 den Widerstand der Parlamentsräte hervor. Da diese ihre Position gefährdet sahen, forderten sie unter anderem ein Mitbestimmungsrecht bei Steuerbeschlüssen und Ämterverkauf. Bei den Auseinandersetzungen Mazarins mit den aufstän-

27 BAYARD, Monde des financiers, S.29f. spricht von einer achtfachen Erhöhung der Steuerlast zwischen 1575 und 1653.

28 Petitfils, Fouquet, S. 58. 
dischen Parlamentsräten, die sich im Frühsommer 1648 zuspitzten und im August in der Fronde parlementaire gipfelten, bewies der neue Intendant von Paris, Nicolas Fouquet, seine Treue zum Premierminister und zur Krone. Durch eine geschickte, die Spaltungen im Parlament vertiefende Politik gelang es ihm, die Gegner der Regierung zu entzweien, die königliche Autorität zu festigen und eine Beruhigung der Lage herbeizuführen. Durch einen Beschluss der Parlamentsräte verlor Fouquet zwar im Zuge der Fronde sein Amt als Intendant, gleichzeitig jedoch förderten die allgemeinen politischen Unruhen sowie Fouquets Einsatz für die Regierung seinen schnellen Aufstieg: 1650 wurde ihm der Kauf des Amtes eines procureur général du parlement de Paris bewilligt ${ }^{29}$. Mit den avocats généraux gehörte er nun zu den Vermittlern zwischen Krone und Parlament und konnte auf beide Seiten gleichermaßen Einfluss ausüben ${ }^{30}$. Für den durch die Fronde in seiner Position stark erschütterten Mazarin war die Schlüsselposition des neuen procureur général von großer machtpolitischer Bedeutung und so hatte er ein großes Interesse, Nicolas Fouquet an sich zu binden. 1651 wurde dieser in den engeren Kreis um den Premierminister aufgenommen ${ }^{31}$. Zugleich verstärkte Fouquet, unterstützt von seinem Bruder Basile und der Marquise du Plessis-Bellière, seine Bemühungen um den Aufbau eines eigenen Netzwerkes von politischen Verbindungen. Die Bildung eines solchen Netzwerkes war im 17. Jahrhundert neben einem festen Familiengefüge unerlässlich für eine politische Karriere. Durch die Anwerbung von Klienten band der Patron Personen aus den verschiedensten Bereichen in einem Treueverhältnis an sich. Die Klienten dienten vor allem als Lieferanten von Informationen und wurden als Gegenleistung für ihre Dienste von dem einflussreichen und wohlhabenden Patron protegiert. Ein Klient konnte entweder durch direkte Kontakte oder mit Hilfe eines courtier, eines Vermittlers, angeworben werden, der seine gesellschaftliche Stellung und sein Vermögen steigerte, indem er seine Klienten in den Dienst eines höheren Patrons stellte ${ }^{32}$. Die Netzwerke durchzogen ganz Frankreich und verbanden die oberen Gesellschaftsschichten miteinander. Sein neues Amt gab Fouquet den Zutritt zu der parlamentarischen Elite und mit ihr wichtige Kontakte. Weitere nützliche Verbindungen versprach seine zweite

29 Der Überschreibungsvertrag für das Amt des procureur général im Parlament von Paris ist nicht überliefert. Es ist jedoch bekannt, dass der Preis 450000 Livres betrug (DEssert, Fouquet, S.373).

30 »Officier partageant avec les avocats du roi ou les avocats généraux les fonctions du ministère public devant les tribunaux. Près des cours souveraines était un procureur général et près des autres juridictions inférieures un procureur du roi, subordonné au procureur général et n'étant en réalité que son substitut. Le procureur général occupait le premier rang dans le parquet des gens du roi« (MARION, Institutions de la France, S. 460).

31 Das Netzwerk des Kardinals wurde vor allem nach der Fronde zu einem der einflussreichsten des Landes und zeichnete sich durch eine relative Geschlossenheit aus; vgl. Petitfils, Fouquet, S. 100.

32 Kettering, Patrons, S.3-5. Siehe auch Kapitel 1: Patrons and Clients, S. 12-29. Zu Fouquet siehe S. 193-195. 
Ehe, die Nicolas im Jahre 1651 mit Marie-Madeleine de Castille (1636-1716) schloss. Seine neue Gattin bot ihm wertvolle Verbindungen zur Hof- und Finanzwelt und damit wichtige Voraussetzungen für den Aufbau eines eigenen Netzwerkes von Beziehungen ${ }^{33}$. In der Folge stellten sich vor allem Parlamentsräte und reiche Financiers ${ }^{34}$ in die Dienste Fouquets und ließen sich, wohl auch aufgrund seines Einflusses auf den Premierminister, von ihm protegieren.

In den Jahren der Fronde des princes und des Exils Mazarins, der nach dem Landesverweis durch das Parlament im Februar 1651 nach Deutschland geflohen war, konnte sich Fouquet, unterstützt durch seine weitreichenden Beziehungen, in seiner schwierigen Stellung zwischen dem Parlament und der Königsfamilie politisch behaupten. Offiziell arbeitete er in der Hauptstadt gegen den Kardinal, blieb Mazarin, der von Brühl aus die Regierungsgeschäfte weiterführte, aber treu ergeben. Mit Hilfe seines Bruders Basile, der seit 1649 in den Diensten des Kardinals stand, informierte er den verbannten Premierminister über das Geschehen in Paris sowie seine Maßnahmen, die Zerstreuung der Mazarinschen Besitztümer zu verhindern. Dadurch erwarb er sich das volle Vertrauen des Kardinals:

Je remercie de tout mon cœur M. le Procureur général de la bonté qu'il a pour moy touchant la main-levée des saisies; je n'en serai jamais ingrat. Je le prie de continuer; car je n'ay qui que ce soit au Parlement qui me donne aucun secours, et faute de cela l'innocence court grand risque d'estre opprimée. Si M. le Procureur général croyait qu'il fallût faire quelque présent à quelqu'un, qui soit capable de faire quelque chose à son avantage, j'en suis d'accord ${ }^{35}$.

Fouquet führte Verhandlungen zwischen dem Königshaus und den frondeurs und machte in zwei Denkschriften Vorschläge zur Überwindung der Krise, die schließlich angenommen wurden ${ }^{36}$. Wenn Mazarin 1653 als triumphaler Sieger nach Paris zurückkehren konnte, hatte er das, neben den militärischen Erfolgen Turennes, den unermüdlichen Bemühungen Fouquets zu verdanken: »Nicolas Fouquet était l'instrument essentiel de la domestication et de l'humiliation du Parlement, brisé dans son ambition de devenir un organe politique $\ll^{37}$. Sein politischer Ehrgeiz, sein weites Netzwerk sowie seine Treue zu Mazarin verhalfen ihm zu einem entscheidenden weiteren Schritt in seiner

\footnotetext{
${ }^{33}$ Marie-Madeleine de Castille war die einzige Erbin einer reichen, in den Adelsstand erhobenen Familie von Kaufleuten und Offizieren mit zahlreichen Verbindungen in den Richterstand und die Finanzwelt. Wichtige Kontakte für Fouquet waren die Cousins seiner Frau: Nicolas de Neufville, maréchal de France und Gouverneur von Ludwig XIV., und Nicolas Jeannin de Castille, trésorier de l'Épargne (vgl. Dessert, Fouquet, S. 77).

34 Dent, Clienteles, S. 52, zählt 116 Financiers zu den direkten Klienten Fouquets (gegenüber 114 in Mazarins und 55 in Colberts Netzwerk).

${ }^{35}$ Mazarin in einem Brief an Basile Fouquet vom 16. Mai 1651, in: Mazarin, Lettres, Bd. IV, S.186.

36 Vgl. ChÉruel, Mémoires, Bd. I, S. 135-138.

37 Petitfils, Fouquet, S. 93.
} 
Karriere. Als am 2.Februar 1653 der surintendant des Finances La Vieuville starb, musste in der noch immer angespannten politischen Lage schnell ein Nachfolger gefunden werden. Nicolas Fouquet stellte sich in einem Brief an Mazarin, in dem er seine Qualitäten hervorhob, als Kandidat zur Verfügung ${ }^{38}$. Der Kardinal, der durch die Fronde den größten Teil seines Vermögens eingebüßt hatte, war an einem loyalen Finanzminister interessiert, der gute Verbindungen zur Welt der Financiers besaß, geschickt zu verhandeln wusste und widerstandslos seinen Geldforderungen nachgab. Er entschied sich, zur besseren Kontrolle und um die Machtfülle zu minimieren, für eine doppelte Besetzung des Amtes und gab die Namen der neuen Finanzminister am 7. Februar 1653 bekannt: Abel Servien und Nicolas Fouquet ${ }^{39}$. Fouquet stand damit im jungen Alter von achtunddreißig Jahren beinahe an der Spitze der Macht in Frankreich. Nur das Amt des Premierministers oder des Kanzlers hätten ihn noch höher steigen lassen können.

\section{Die ersten Jahre des surintendant des Finances Nicolas Fouquet (1653-1655)}

Das Amt des surintendant des Finances war seit seiner Einrichtung im Jahre 1552 durch Heinrich II. Veränderungen unterworfen, die bis zur Auflösung der surintendance reichten. Heinrich IV. ersetzte den Finanzminister 1594 durch einen zentralen Finanzrat von acht Mitgliedern, doch schon wenige Jahre später wurde die surintendance de facto wieder eingerichtet. Nach ihrer rechtlichen Wiederherstellung im Jahre 1611 blieb sie ein ständiges Amt, welches entweder von einer oder von zwei Personen bekleidet wurde ${ }^{40}$. Unter Mazarin wurde sie zu einem der ersten Ämter des Staates; der Finanzminister kontrollierte als ordonnateur principal des fonds alle Vorgänge der Finanzverwaltung im Königreich, verfügte uneingeschränkt über die Finanzen des Königs und musste nur diesem Rechenschaft ablegen. In ihren Aufgaben wurde die surintendance unterstützt von drei Organen der Finanzverwaltung: den ordonnateurs, die als Anweisungsberechtigte bei der Vergabe und der Erhebung von Finanzmitteln beteiligt waren, den comptables, die als Buchhalter die Verwaltung der königlichen Einnahmen und Ausgaben überwachten, so-

38 In dem Brief an Mazarin rühmt Fouquet neben seiner politischen Erfahrenheit seine Rechtschaffenheit, Treue und seine exzellenten Verbindungen in die Finanzwelt (Fouquet an Mazarin in einem Brief vom 2. Januar 1653, Arch. Aff. étr., M.D. France, 892, fol. 39f., nach: Antoine, Cœur de l'État, S. 248).

39 Pouvoir des surintendants daté de Paris le 8 février 1653 (AN O 110 , fol. 23 f.).

40 Von 1624-1626, 1632-1640, 1643-1647, 1649-1650 sowie von 1653-1659 besetzten jeweils zwei surintendants des Finances das Amt (vgl. BARBICHE, »Surintendants des finances «, in: DGS, S.1493). Petrtfils, Fouquet zitiert auf S. 127 zur Erläuterung des Grundes für eine Doppelbesetzung Kardinal de Richelieu: »Deux hommes piqués de jalousie ne s'entendront jamais pour piller les deniers de l'Epargne!« 
wie den contrôleurs, die die Richtigkeit der Vorgänge überprüften und diese in Rechnungsbüchern festhielten ${ }^{41}$. Fouquet übernahm das Amt mit Abel Servien zu einem Zeitpunkt, als das Finanzsystem zwar auf den ersten Blick stabil schien, sich in Wirklichkeit aber der Zusammenbruch der Finanzverwaltung anbahnte. Infolge der langen Kriegsjahre und der hohen Ausgaben für die Armee sowie der steigenden Hofhaltungskosten waren die Staatskassen nahezu leer. Dem drohenden Staatsbankrott und dem beständigen Druck durch Hof und Premierminister versuchte die Finanzverwaltung über alle Organe hinweg mit semilegalen Maßnahmen zu begegnen. Man begann, die gebräuchlichsten Zahlungsmittel der königlichen Staatskasse, die mandements und die billets de l'Épargne, die einem königlichen Gläubiger mit unterschiedlicher Sicherheit die Auszahlung der jeweiligen Geldsumme durch einen königlichen Beamten zusicherten ${ }^{42}$, über das laufende Jahr hinaus für die kommenden Jahre auszustellen und somit die Staatseinnahmen im Voraus auszugeben. Für die Financiers, die sich im Normalfall durch hohe Zinssätze an der Misere des Staates bereicherten, bedeuteten die Staatsanleihen in Krisenzeiten ein hohes Risiko: Wegen der geringen Steuereinnahmen und der überhöhten Zinssätze wurden die Schulden oft nicht zurückgezahlt. Die häufig nahezu wertlosen Schuldscheine konnten von den Angestellten der Finanzverwaltung zu Spottpreisen gekauft werden, die sie dann nach einer Neubewertung durch die Buchhalter mit einem enormen Gewinn einlösten. Ähnlich wurde mit bereits ausgezahlten Wechseln verfahren, die durch Neubewertungen auf Kosten der Staatskasse von den königlichen Beamten bis zu vier Mal eingelöst wurden. Diese Maßnahmen führten, anstatt zu einer Entspannung der finanziellen Lage, zu einer Lähmung der Finanzmaschinerie und einer Zunahme der Staatsverschuldung, von der einzig die an der Hintergehung des Fiskus Beteiligten profitierten ${ }^{43}$.

Als Fouquet zusammen mit Servien 1653 die surintendance des Finances übernahm, waren sämtliche Einnahmemöglichkeiten aus gewöhnlichen Steuern, Renten und dem Verkauf von Ämtern bereits für mehrere Jahre ausgeschöpft. Die finanzielle Situation Frankreichs schien aussichtslos. Durch die Neubewertung des Livre im Juli 1653 konnte man eine kurzfristige Entspan-

${ }^{41}$ Eine detaillierte Darlegung der Zusammensetzung der einzelnen Organe findet sich bei Dent, Crisis, ab S. 243 beziehungsweise Antorne, Cœur de l'État, ab S.221.

${ }^{42}$ Die mandements waren die gebräuchlichere Form der vom Staat ausgestellten Schuldscheine. Sie wiesen einen bestimmten königlichen Beamten an, dem Eigentümer des Scheines die auf dem Schriftstück genannte Summe auszuzahlen. Der Beamte zog den Betrag von den jährlichen Einnahmen durch direkte Steuern ab, die er an die Staatskasse $\mathrm{zu}$ senden hatte. Ein billet de l'Épargne war ein dem mandement ähnliches Zahlungsmittel, doch gab es den Gläubigern weniger Sicherheit. Mit diesem Schuldschein erkannte der Aussteller an, dass der Scheineigner dem Staat eine bestimmte Summe geliehen hatte, die ihm von der königlichen Kasse zurückgezahlt werden sollte. Ein Datum sowie die Auszahlungsstelle wurden nicht genannt, es erfolgte also keine genaue Anweisung an einen königlichen Beamten, das geliehene Geld zurückzuzahlen (vgl. DENT, Crisis, S. 244f.). ${ }^{43}$ Ibid., S. 245f. 
nung der Lage herbeiführen, im September 1654 schien der Bankrott jedoch wieder in unmittelbare Nähe gerückt. In dieser Krisensituation zeigte sich Servien, der alle Vorschläge zur Besserung der Lage ablehnte, starrköpfig und unflexibel. Fouquet, der stattdessen Reformvorschläge präsentierte, profitierte von dieser Entwicklung. Sie erlaubte ihm, weitere Klienten an sich zu binden und durch ein règlement écrit vom 24. Dezember 1654 die Zuständigkeitsbereiche der beiden Finanzminister zu trennen - Servien überwachte fortan die Ausgaben, Fouquet war für die Einnahmen der Krone verantwortlich ${ }^{44}$. Damit hatte er von nun an zwar die aufwendigere, aber auch die einflussreichere Position im Finanzbereich inne.

Im Vordergrund des Fouquetschen Reformplanes stand eine neue Art des Umganges mit den Financiers, auf die man angewiesen war, wollte man den Staatsbankrott verhindern. Vor allem sollte ihr Vertrauen in die Zahlungsfähigkeit der Krone wiederhergestellt werden. Gerüchte um einen drohenden Bankrott mussten zerstreut, aller Argwohn vermieden werden. Die Krone sollte sich großzügig zeigen, da die Financiers nur dann bereit waren, ein Risiko einzugehen, wenn sie mit einem Gewinn rechnen konnten ${ }^{45}$. Um das Vertrauen der königlichen Gläubiger wiederherzustellen, war Fouquet zunächst auf kurzzeitige Darlehen angewiesen und erhielt Hilfe aus dem Kreis seiner Freunde, Verwandten und Klienten. Damit zeichnete sich schon früh eine Besonderheit des Fouquetschen Finanzsystems ab. Um die immensen Geldforderungen der Armee und des Hofes befriedigen zu können, wurde der surintendant Fouquet zu einem privaten Bankier der Krone, der Anleihen auf seinen Namen aufnahm, seine Klienten und seine Familie als Gläubiger verpflichtete und neben seinem eigenen auch das Vermögen seiner Frau und Familie verpfändete. Seine persönlichen Verflechtungen mit den Finanzen des Königreiches gingen so weit, dass er in besonderen Fällen sein Eigentum verkaufen musste, um die Kriegskosten bezahlen zu können, deren Bestreitung zeitweise fast vollständig von seiner persönlichen Liquidität abhing. Fouquet brachte damit nicht nur seine eigene Familie und Freunde in Gefahr ${ }^{46}$, er verlagerte zudem die Beschaffung öffentlicher Mittel auf eine kleine Anzahl von Personen und sorgte somit nicht für eine Besserung der allgemeinen Finanz-

44 Règlement écrit: BN Cinq Cents Colbert 194, fol.289r-290v. Siehe auch: FouQuET, Euvres, Bd.V, S.353. Zu den Auseinandersetzungen zwischen Servien und Fouquet in der Führung des Finanzapparates siehe: Antoine, Cœur de l'État, S. 260.

$45 »$ Flatter [des gens d'affaires] et, au lieu de leur disputer des intérêts et profits illégitimes, leur faire des gratifications et indemnités de bonne foi quand ils avaient secouru à propos, et que le principal secret, en un mot, était de leur donner à gagner, étant la seule raison qui fait que l'on veut bien courir quelque risque; mais surtout de s'établir la réputation d'une sûreté de parole si inviolable, qu'on ne croit pas même courir aucun danger « (Fouquet, Euvres; zitiert nach: Cornette, Chronique, S. 28).

46 Fouquet, Euvres, Bd. V, S.67. LaIR, Fouquet, Bd. I, S. 441 zitiert hierzu Isaac Bartet, einen Zeitgenossen Fouquets: »S'il fut mort, il laissait sa maison et tous ses amis dans une pauvreté ridicule«. 
lage. Darüber hinaus führten die kurzfristigen Anleihen, die Fouquet im Namen der Krone bei den Gläubigern aufnahm, zu einem enormen Anstieg der Zinssätze, die von den Financiers, welche sich ihrer überlegenen Position bewusst waren, in die Höhe getrieben wurden: Lag die offizielle Grenze der vom König bezahlten Zinsen bei 5,5\%, so stiegen sie zwischen 1653 und 1661 auf bis zu 50\% an ${ }^{47}$. Der surintendant, der nur unzureichend versuchte, die bestehenden Betrügereien zu unterbinden, wurde damit zum Kollaborateur der Financiers ${ }^{48}$. Nach Petitfils sei sich Fouquet des Ausmaßes der Finanzmisere nicht bewusst gewesen, ein Umstand, den der Biograph auf die mangelnde Erfahrung des surintendant in Finanzdingen zurückführt ${ }^{49}$. Auch Dent zieht in seiner Betrachtung über die Krise in der Finanzverwaltung zwischen 1653 und 1661 den Schluss, der surintendant sei weit davon entfernt gewesen, über die Finanzen des Königs zu verfügen. Vielmehr habe er sich dem bestehenden System angepasst, ohne es in Frage zu stellen, und seinem ersten Sekretär, dem 1654 eingesetzten Jacques Amproux Delorme, in allen Fragen vertraut ${ }^{50}$. Dieser gewann unter Fouquet eine ungeheure Machtfülle, welche er zu seinem eigenen Nutzen einzusetzen wusste: Er stellte falsche Schuldscheine aus, veruntreute Gelder, verteilte hohe Zuwendungen unter seinen Helfern und bereicherte sich selbst in hohem Maße an den öffentlichen Geldern. Seine Bestrebungen reichten bis hin zu einem Versuch im Jahre 1657, Fouquet zu stürzen, was aber durch den Finanzintendanten Barthélemy Hervart und den Schatzmeister Nicolas Jeannin de Castille verhindert werden konnte und zur Absetzung Delormes führte ${ }^{51}$. Doch auch bei dessen Nachfolger Louis Bruant änderte Fouquet nichts an diesem Abhängigkeitsverhältnis; genauso wenig erfolgte eine Reform innerhalb der Finanzverwaltung, die der Korruption und Misswirtschaft ein Ende gesetzt hätte.

Neben den Ansprüchen von Hof und Armee, denen Fouquet gerecht zu werden suchte, wurde der Finanzminister von seinem eigenen Patron, dem Kardinal Mazarin, unter Druck gesetzt. Dieser hatte trotz der Bemühungen Fouquets während der Fronde nahezu sein gesamtes Vermögen verloren und versuchte nun, seine Kassen wieder zu füllen. Unterstützt wurde er bei diesen Bemühungen von seinem engsten Vertrauten Colbert, der damit beauftragt war, die verlorenen Güter des Mazarinschen Hauses wiederzuvereinen. Auch Fouquet, der dem Kardinal sowohl seinen eigenen als auch den Aufstieg seiner Brüder und Freunde verdankte, konnte sich den Wünschen Mazarins nicht entziehen und war täglich neuen finanziellen Begehrlichkeiten ausgeliefert: »Je suis très obligé de M. le procureur général de la manière dont il

47 Vgl. Dent, Crisis, S. 248.

${ }^{48}$ Um die Situation zu entschärfen, führte Fouquet 1656 eine neue Steuer ein, die von den Gläubigern an die Krone gezahlt werden musste.

$49 \mathrm{Vgl}$. PetitfiLs, Fouquet, S. 146.

50 Vgl. Dent, Crisis, S.248f.

51 Ibid., S. 249. 
en use et pour ce qui regarde le service du roi et pour mes intérêts particuliers $\ll^{52}$.

Für Mazarin, der nur über einen geringen Einfluss auf die Finanzwelt verfügte, war die Unterstützung durch Fouquet unerlässlich und ein Grund dafür, dass der surintendant trotz zunehmender Spannungen mit Colbert nicht in Ungnade fiel.

\section{Die Jagd der Natter Spannungen in der Regierungselite (1655-1661)}

Das anfänglich gute Verhältnis zwischen Fouquet und Colbert ${ }^{53}$ bekam bereits 1651, ein Jahr nach ihrem ersten Zusammentreffen, sichtbare Risse, als sich Fouquet bei dem Intendanten des Premierministers im Interesse seines Bruders Basile schriftlich über Mazarin beschwerte. Colbert, dessen Verhältnis zu Basile seit jeher getrübt war, gab den Brief an Mazarin mit folgendem Begleitschreiben weiter und denunzierte damit Nicolas Fouquet:

La mauvaise conduite que l'on a tenue en vos affaires vous a réduit à ce point de nécessité que vous avez besoin de tous ces gens-là, et que plus vous en avez besoin, plus ils vous tiennent le pied sur la gorge pour exiger de vous des choses que vous n'êtes pas en état ni en pouvoir de leur accorder. Il [Nicolas] est de mes amis, et je suis obligé de vous dire qu'il vous a bien servi depuis que j'ai la direction de vos affaires. Je ne puis toutefois m'empêcher de blâmer son procédé et de le trouver tout à fait extraordinaire ${ }^{54}$.

Ob Colbert mit diesen Worten den Unmut Mazarins gegenüber Fouquet entzündete oder Mazarin seinem Finanzminister bereits zuvor kein volles Vertrauen geschenkt hatte, ist unklar.

Die Spannungen innerhalb des Kreises um den Kardinal verschärften sich vor allem nach der Ernennung Fouquets zum Finanzminister, als Colbert, unterstützt von Le Tellier, begann, Zweifel an Fouquets Eignung zu äußern. Im

52 Zitiert nach: ChÉruel, Mémoires, Bd. I, S. 281 f.

53 Um im Jahre 1650 das Amt des procureur général de Paris zu erhalten, bemühte sich Nicolas Fouquet um die Protektion Michel Le Telliers, der die von Gaston d'Orléans vorgebrachten Einwände gegen seine Ernennung zerstreuen sollte. Fouquet fand die Unterstützung Colberts, der zu diesem Zeitpunkt noch als Sekretär in Diensten des secrétaire d'État à la guerre, Michel Le Tellier, stand. Colbert bat in einem für die Zeit typischen Brief um die Unterstützung Fouquets durch Le Tellier: »Monsieur Fouquet, qui est icy venu par ordre de Son Eminence, m'a desjà témoigné trois fois différentes qu'il avoit une très forte passion d'estre du nombre de vos serviteurs particuliers et amis par une estime très particulière qu'il fait de vostre mérite; et qu'il n'avoit point d'attachement particulier avec aucune autre personne qui luy pût empêcher de recevoir cet honneur [...]. J'ai cru qu'il était bien à propos, estant homme de naissance et de mérite particulier et en estat mesme d'entrer un jour dans quelque charge considérable, de luy faire quelques avances de la mesme amitié de votre part« (Colbert, Lettres, Bd.I, S.24f., Brief vom 9. August $1650)$.

54 Brief Colberts an Mazarin vom 10. November 1651, in: ibid., S.165f. 
Gegensatz zu vielen seiner Vorgänger, wie beispielsweise dem Finanzminister der Jahre 1649-1650, Particelli d'Émery, der mit einer zwanzigjährigen Erfahrung im königlichen Finanzwesen die surintendance übernommen hatte, war Fouquet nicht mit dem komplexen Finanzsystem vertraut und trat sein Amt nahezu unvorbereitet und in einem ungewöhnlich jungen Alter an. Mazarin reagierte zunächst nicht auf die Vorwürfe. Erst als Fouquet die beständigen Forderungen des Kardinals nach immer höheren Geldsummen für seinen persönlichen Bedarf wegen der schwierigen politischen und finanziellen Lage nicht mehr umgehend befriedigen konnte, begann er seinen Unmut zu äußern ${ }^{55}$.

Auch Fouquets Bruder Basile wurde alsbald in den Konflikt hineingezogen. Der Ausbau der Fouquetschen chaîne de fidélité rief das Misstrauen des Kardinals hervor, der damit die Unabhängigkeit der Fouquets gestärkt sah. Bereits 1655 beschwerte sich der Kardinal bei Basile darüber, dass dieser zusammen mit seinem Bruder hinter seinem Rücken seine treuesten Anhänger abwürbe. Im Juni 1657 spitzten sich die Unstimmigkeiten zwischen dem Kardinal und Nicolas Fouquet zu: Mazarin warf seinem Protegé vor, er bringe große Summen für die Armee auf, ohne seinen anderen Verpflichtungen nachzukommen ${ }^{56}$. Nicolas, der die Vorwürfe zwar zu entkräften wusste, bangte fortan um seine Stellung. In seiner Verteidigungsschrift, die er zwischen 1661 und 1664 verfasste und die 1696 veröffentlicht wurde, macht der Finanzminister deutlich, dass er 1657 fürchtete, Mazarin könne seine Absetzung im Sinn haben: »[En 1657], je fus convaincu qu'on me vouloit perdre, \& conçus une grande douleur, de ce que tant de services, tant de soins, de travaux, de risques, n'avoient pas d'autre reconnaissance $\ll^{57}$. Im Sommer 1657 verfasste der Finanzminister ein Dokument, das den Bruch zwischen Mazarin und Fouquet verdeutlicht: der Entwurf eines Notfallplanes, der nach seinem späteren Fundort »Plan von Saint-Mandé« genannt wurde ${ }^{58}$. Er enthält Anweisungen

55 Der immerwährende Druck, den Mazarin auf den Oberfinanzminister ausübte, kommt in seinen Briefen zum Ausdruck: »Il y a deux petites affaires que M. le procureur général m'avait promis de me faire rendre, mais il l'aura oublié « (Brief Mazarins an den Abbé Fouquet vom 16. Mai 1657, in: BN ms. fr. 23202, fol. 223v.).

56 Siehe: Bibliothèque de l'Institut, Collection Godefroy, ms. 548, fol. 591, Brief Mazarins an Basile Fouquet vom 4. Juli 1657.

57 Fouquet, CEuvres, Bd. V, S. 78. In Bd.X, S. 38 bezieht er seine Befürchtungen ausdrücklich auf eine Initiative Mazarins: »Voilà les motifs sur lesquels estoit fondée la juste crainte que j'avois, qu'un Ministre tout-puissant n'entreprist ma perste sous des pretextes specieux, dont on ne manque jamais contre ceux qui ont eu quelque employ dans les Finances $\ll$.

58 Der Plan von Saint-Mandé in der Fassung von 1657 ist abgedruckt in: DesserT, Fouquet, S.354-364. In seiner Verteidigungsschrift beteuert Fouquet an verschiedenen Stellen die Skizzenhaftigkeit des Plans, der aus einem Affekt und ohne die Absicht einer Umsetzung entstand. Dies erklärt er damit, dass der Plan weder vervielfältigt noch an die betroffenen Personen verteilt wurde (die nicht einmal mündlich von ihm in Kenntnis gesetzt wurden) und auch nicht an einem sicheren Ort Aufbewahrung fand. Siehe unter anderem: Fouquet, Euvres, Bd. II, S. 48-62; Bd. VII, S.339f.; Bd. X, S. 17, $73 f$. 
für den Fall einer Verhaftung Fouquets, führt die handelnden Personen namentlich auf, ohne jedoch einen Adressaten zu nennen, der die Ausführung hätte leiten können. Wie Fouquet in seiner Verteidigungsschrift erklärt, ist es ein Plan, der nicht aus Misstrauen gegen das königliche Haus, sondern allein aus den Befürchtungen vor den Handlungen Mazarins entstand ${ }^{59}$. In der Präambel legt der Finanzminister die Gründe für seinen Argwohn gegenüber dem Kardinal dar. Er beschreibt dessen eifersüchtiges Naturell, sein ungerechtes Wesen und den gefährlichen Charakter Mazarins, der wie ein absoluter Herrscher auch über den König und die Königin regiere: »Il faut donc craindre tout et le prévoir, afin que si je me trouvais hors de la liberté de m'en pouvoir expliquer « ${ }^{60}$. Der Plan enthält zwar keinen offenen Aufruf zu einer Revolte, zeigt jedoch, dass Fouquet im Falle seines Sturzes davon ausging, Freunde und Verwandte würden sich für ihn einsetzen und Truppen mobilisieren, ohne an eigene Interessen zu denken. Fouquet gibt Instruktionen für den Ernstfall, wobei er mehrere Krisensituationen beschreibt. Im Falle seiner alleinigen Verhaftung sollte sein Bruder Basile die Leitung der Familie übernehmen und die nötigen Schritte zu seiner Befreiung in die Wege leiten. Interessanter ist die zweite von Fouquet dargestellte Krisensituation: Sollte Basile ebenfalls verhaftet werden, beide aber die Erlaubnis erhalten, sich zu sehen sowie Besuche zu empfangen, so könnten sie gemeinsam die nötigen Anweisungen geben. In den ersten Monaten sollte der Kontakt zu Freunden aufgenommen werden, ohne dass ernste Maßnahmen zur Rettung der Gefangenen ergriffen würden. Nach drei Monaten sollten sich die Vertrauten und Freunde Fouquets nach den Anweisungen Mme du Plessis-Bellières um die Aufstellung von Truppen an verschiedenen, strategisch günstigen Punkten des Königreiches bemühen und im Falle eines Prozesses gegen Fouquet die Freilassung des Gefangenen beantragen sowie sein Vermögen und seine Papiere in Sicherheit bringen. Der Plan bricht unvermittelt ab. Sein fehlerhafter Stil sowie die Unvollständigkeit der Sätze zeigen, dass er in Eile und Erregung nur als Skizze niedergeschrieben wurde. Einen geplanten Aufstand gegen die Krone, wie Fouquet später vorgeworfen wurde, skizziert er nicht. Er macht jedoch deutlich, dass sich Nicolas Fouquet seiner politisch unsicheren Lage bewusst war.

Den ersten Zerwürfnissen mit dem Kardinal folgte wenig später ein weitaus persönlicheres: der Bruch des Finanzministers mit seinem Bruder Basile, der das feste Familiengefüge der Fouquets ins Wanken brachte ${ }^{61}$. Seit langem hatte es zwischen den Brüdern Unstimmigkeiten gegeben. Basile warf dem Älteren vor, sich zu unabhängig zu gebärden, obwohl er das Amt des Finanz-

59 Ibid., Bd.X, S. 38.

60 Projet de Saint-Mandé, abgedruckt in: Dessert, Fouquet, S. 355.

61 FouQuet, Euvres, Bd.X, S.13: »Il est notoire que mon frere l'Abbé \& moy nous avions esté toujours en tres-bonne intelligence, \& que nostre grande brouillerie arriva environ le mois de Decembre 1657 «. 
ministers allein seiner Fürsprache beim Kardinal verdanke ${ }^{62}$. Nicolas missbilligte zum einen die Eskapaden seines Bruders, die die ganze Familie in Misskredit brächten ${ }^{63}$. Zum anderen warf er ihm vor, hinter seinem Rücken den Einfluss auf den Kardinal zu missbrauchen. Die Anschuldigungen steigerten sich bis zu offenen Hassausbrüchen, und Ende 1658 kam es zum endgültigen Zerwürfnis ${ }^{64}$. Damit war Fouquets Stellung erheblich geschwächt, denn der Bruder begann, das gemeinsam aufgebaute Netzwerk durch aktive Abwerbung zu zerstören. Zudem denunzierte er Nicolas bei Mazarin und verbündete sich mit Delorme, dem 1657 entlassenen ersten Sekretär des Finanzministers, um mit dessen Hilfe dem Ruf Nicolas' zu schaden ${ }^{65}$. Fouquet, von den familiären Zerwürfnissen schwer getroffen, musste nun nicht nur im beruflichen Umfeld, sondern auch auf der seine Politik stützenden privaten Ebene um seine Machtposition bangen. Indem er ihnen Ämter zuschanzte und Geldgeschenke machte, versuchte er, die ihm weiterhin ergebenen Klienten des gemeinsamen Netzwerkes an sich zu binden, musste jedoch erkennen, dass einige Protagonisten des Plans von Saint-Mandé auf der Seite Basiles standen. Die Konsequenz war eine Überarbeitung des Plans im Jahre 1659: Fouquet ordnete an, im Falle seiner eigenen Verhaftung auf den Bruder Basile und dessen Handlungen ein besonderes Augenmerk zu richten. Zudem nahm die soeben gekaufte bretonische Insel Belle-Île einen besonderen Platz als Stützpunkt für Truppen und Schiffe ein ${ }^{66}$. Wie bereits die erste Fassung des Plans von Saint-Mandé, so wurde auch die zweite Version in großer Erregung verfasst. Nicolas versteckte den Plan hinter einem Spiegel, wo er 1661 von königlichen Beamten entdeckt wurde.

Doch weder die Unstimmigkeiten mit Mazarin noch der Bruch mit dem Bruder Basile stellten für die politische Karriere Fouquets eine wirkliche Gefahr dar; die hauptsächliche Bedrohung kam von Colbert, der unentwegt versuchte, dem Finanzminister zu schaden. Die >Jagd der Natter ${ }^{67}$ drohte im Jahr 1659 erstmalig Erfolg zu haben: Nach Abel Serviens Tod am 16. Februar überzeugte Colbert den Kardinal, dass dieser selbst das frei gewordene Amt besetzen sollte, um die Kontrolle über die Finanzverwaltung zu erlangen.

62 Gourville, Mémoires, S. 109.

63 Gemeint ist hier die lange und Aufsehen erregende Beziehung des Abbés zur Duchesse de Châtillon.

64 Patin, Correspondance, S.354 in einem Brief an Falconet vom 28. Januar 1661: »Comme il [Nicolas Fouquet] était dans l'antichambre du cardinal Mazarin, son frère l'abbé Fouquet y survint (ils sont mal ensemble il y a deux ans): ils commencèrent à se quereller l'un et l'autre, en présence de beaucoup de monde«.

65 Fouquet, Euvres, Bd. X, S. 15, 19.

66 Fouquet kaufte Belle-Île, die größte Insel der Bretagne, Ende 1658 auf Drängen Mazarins und mit der ausdrücklichen Zustimmung des Königs, um damit den Kauf der Insel durch den königsfernen Louis de Cossé-Brissac zu verhindern. Er nutzte die Insel in der Zukunft als Ausgangspunkt für seine überseeischen und kolonialen Aktivitäten und begann 1659 mit dem Bau einer Residenz sowie einer Festungsanlage auf der Insel.

67 Die Natter ist das Wappentier Colberts. 
Fouquet wusste dies abzuwenden, indem er dem Premierminister die damit verbundene Schwächung seiner eigenen Position vor Augen führte, die einer Verschlechterung der allgemeinen Finanzlage folgen würde. Mazarin, dessen Vermögen längst den Stand vor der Fronde übertraf, dem aber gleichwohl nichts ferner lag, als die Finanzlöcher des Staates aus eigener Tasche zu stopfen, ernannte Fouquet am 21. Februar zum alleinigen Finanzminister ${ }^{68}$.

Colbert verstärkte in der Folge seine Bemühungen, die Absetzung Fouquets zu erreichen. Die Beschwerden über Missstände in der Finanzverwaltung häuften sich, und Colbert ging so weit, in einem geheimen Dossier an Mazarin alle Vorwürfe gegen den surintendant genau darzulegen und einen ausführlichen Reformplan zur Beseitigung der Finanzmisere vorzulegen, der den Sturz Fouquets und die Einrichtung einer chambre extraordinaire für seine Verurteilung vorsah ${ }^{69}$. Doch auch in diesem Fall kam ihm Fouquet, der die Post Mazarins seit langem abfing und somit über Colberts Pläne informiert war, zuvor: Er reiste nach Saint-Jean-de-Luz, wo der Premierminister mit den Spaniern über die Beendigung des Krieges verhandelte, und sprach selbst mit dem Kardinal über die missliche Lage der königlichen Finanzen, erarbeitete mit ihm ein Reformprogramm und zeigte sich enttäuscht darüber, dass das persönliche Verhältnis zu dem Vertrauten Colbert getrübt war ${ }^{70}$. Mazarin war offenbar zufrieden mit den Vorschlägen seines Finanzministers, die durch Reformen in der Steuerpolitik und eine verstärkte Einbeziehung der französischen Kolonien in die Handelstätigkeit Frankreichs eine Besserung der Finanzlage versprachen. Er legte seinem Finanzminister nahe, sich mit Colbert auszusprechen und die vorhandenen Spannungen zu beseitigen ${ }^{71}$. Die Aussprache erfolgte kurz nach Fouquets Rückkehr, doch die scheinbare Besserung der Beziehungen zwischen den beiden war nur von kurzer Dauer. Zwar konnte der surintendant des Finances - nach dem Pyrenäenfrieden vom 7. November 1659 - im Januar 1660 erste Reformmaßnahmen durchführen, doch stellte sich damit keine Beruhigung der innenpolitischen Auseinandersetzungen und Anschuldigungen ein. Im Laufe des Jahres begannen sich die Beschwerden Colberts über die Finanzpolitik wieder zu mehren. Der im Sommer 1660 schwer erkrankte Mazarin schenkte den Anschuldigungen erneut ein offenes Ohr.

Ende 1660 erhielt Fouquet von einer Spionin im Umkreis der Königinmutter einen Bericht, der ihm vor Augen führte, dass seine Stellung auch am königlichen Hof gefährdet war: »La Reyne mère dit dimanche dernier sur vous, que M. le Cardinal avoit dit au Roy que si l'on pouvoit vous oster les basti-

\footnotetext{
${ }^{68}$ Fouquet, CEuvres, Bd. XIV, S.237f.

69 Gourville, Mémoires, S.115.

70 Siehe: Brief Mazarins an Colbert vom 21.Oktober 1659, in: Colbert, Lettres, Bd.I, S.514.

71 Siehe: Brief Mazarins an Colbert vom 22. Oktober 1659, in: ibid., S. 517.
} 
ments et les femmes de la teste, vous seriez capable des [plus] grandes choses; mais que surtout il fallait prendre garde à vostre ambition « ${ }^{72}$.

Obgleich Fouquets Stellung bis 1661 überaus instabil blieb, wurde der Finanzminister - neben dem Maréchal de Villeroy, Michel Le Tellier und Zongo Ondedei - zu einem Favoriten um die Nachfolge des kranken Premierministers. Als Finanzminister war Fouquet ein natürlicher Kandidat für die Nachfolge Mazarins - des mächtigsten Mannes im Königreich. Mit der Schwächung der französischen Monarchie durch die Ermordung Heinrichs IV. im Jahre 1610 hatte sich hier ein festes Herrschaftsmuster herausgebildet: Die Regierung wurde während der Minderjährigkeit Ludwigs XIII. an den Premierminister Richelieu übertragen, der diese auch nach der Volljährigkeit Ludwigs in seinen Händen behielt. Gleiches wiederholte sich im Falle Ludwigs XIV. Nach seiner Krönung am 7. Juni 1654 blieben die Regierungsgeschäfte in den Händen seines Premierministers Mazarin. Alles deutete darauf hin, dass sich auch in Zukunft nichts an der Machtverteilung im Königreich ändern sollte und sich der König weiterhin auf einen starken Premierminister an seiner Seite verlassen wollte. So schien es nicht ungewöhnlich, dass der Finanzminister auf eben diese Position im Staat spekulierte.

\section{Der Tod Mazarins und seine Folgen (1661)}

Der prekäre Gesundheitszustand Mazarins verlangte im März 1661 Vorkehrungen für die Leitung des Königreiches nach seinem Ableben. Wenige Tage vor seinem Tod rief der Kardinal Ludwig XIV. und seine drei Minister zu sich, um dem König die Vorzüge der drei darzulegen. An Fouquet lobte er dessen exzellente Fähigkeiten auf dem Gebiet der Finanzen sowie die von ihm in allen Angelegenheiten erteilten ausgezeichneten Ratschläge. Colbert zufolge riet Mazarin jedoch bereits einen Tag später, am 8. März, dem König, auf die Ambitionen seines surintendant achtzugeben. Zugleich legte er dem jungen Herrscher nahe, sich Colberts Unterstützung zu bedienen: »Je vous dois tout, Sire; mais je crois m'acquitter en quelque manière en vous donnant Colbert «73. Noch am selben Tag ernannte Ludwig Colbert zum intendant des Finances. Offiziell sollte er die von der surintendance bis 1659 geschlossenen Verträge überprüfen. De facto erlaubte das neue Amt ihm jedoch einen Einblick in die undurchschaubaren Interna des Finanzsektors und die Arbeitsweise des Finanzministers, die der junge König aufzudecken hoffte ${ }^{74}$.

\footnotetext{
72 Abgedruckt in: ibid., Bd. II, S. XII.

73 Cholsy, Mémoires (2005), S. 118.

74 Ibid., S. 125: »Après avoir mis Colbert dans le conseil des finances pour examiner de plus près la conduite de Fouquet, dont l'heure n'était pas encore venue, il [le roi] songea à la distribution des bénéfices«. Siehe auch: Dessert, Argent, S. 296.
} 
Am 9. März 1661 starb Mazarin. Nur einen Tag später verkündete der König, dass er höchstpersönlich das Amt des Verstorbenen übernehmen werde. Die Frage um Mazarins Nachfolge erübrigte sich somit. Fouquet, dem nahegelegt wurde, mit dem neuen Intendanten der Finanzen zusammenzuarbeiten, konnte darin die beginnende Schwächung seiner Stellung erahnen. Sein Ansehen bei dem jungen Herrscher suchte er mit dem Geständnis zu bessern, dass in der Finanzverwaltung nicht immer nach den Vorschriften gehandelt worden war, versicherte jedoch, dass nichts ohne die Zustimmung des Kardinals geschehen sei. Fouquet versprach dem König, sich seinen Befehlen zu fügen »[et] de servir à l'avenir avec tout le zèle et l'affection imaginables «75. Der König zeigte sich voller Gnade und verzieh dem Minister.

In den ersten Wochen nach Mazarins Tod gelang es Fouquet scheinbar, seine Position in der Regierung zu stärken. Nach den Worten des niederländischen Botschafters Conrad von Beuningen stand Fouquet »à la tête des trois qui composent le Conseil privé. Je vous laisse à penser quelle influence cela peut avoir, non seulement sur les affaires journalières, mais sur tout le cours de nos négociations « ${ }^{76}$. Fouquet begann sowohl im Außenhandel wie in der Außenpolitik eine wichtige Rolle zu spielen. Im April wurde er vom König mit der Gründung eines conseil du commerce beauftragt, zugleich vertraute ihm Ludwig einige geheime diplomatische Missionen an, die der Minister mit Erfolg ausführte 77 . Doch der Aufstieg Fouquets währte nicht lange. Die schlechte finanzielle Lage des Königreichs, dessen Einnahmen bereits für die kommenden zwei Jahre verbraucht waren, warf einen Schatten auf die Beziehung des jungen Monarchen zu seinem Finanzminister. Colbert, der den König wöchentlich über die Finanzen informierte, behauptete zudem, dass Fouquet gefälschte Rechnungen vorlege. Seine Anschuldigungen fanden Gehör. Ludwig, bereits durch die Worte des sterbenden Mazarin argwöhnisch gemacht, zeigte sich zunehmend misstrauisch gegenüber dem Ehrgeiz sowie dem prunkvollen Lebensstil seines Ministers:

La vue des vastes établissements que cet homme avait projetés, et les insolentes acquisitions qu'il avait faites, ne pouvaient qu'elles ne convainquissent mon esprit du dérèglement de son ambition. [...] Mais ce qui le rendait plus coupable envers moi, était que bien loin de profiter de la bonté que je lui avais témoignée en le retenant dans mes conseils, il en avait pris une nouvelle espérance de me tromper, et bien loin d'en devenir plus sage, tâchait seulement d'en être plus adroit. Mais quelque artifice qu'il pût pratiquer, je ne fus pas longtemps sans reconnaître sa mauvaise foi ${ }^{78}$.

Der junge König verabscheute das Gefühl, betrogen zu werden. Am 4. Mai fiel die Entscheidung, Fouquet aus seinem Amt zu entfernen ${ }^{79}$. Wieder ein-

75 BN ms. fr. 7621, fol. 121v.

${ }^{76}$ Conrad von Beuningen in einem Brief an Jan van Witt vom 1. April 1661, zitiert nach: Petitfils, Fouquet, S. 328.

77 Genaue Informationen zu den auswärtigen Missionen: siehe ibid., S.329f.

78 Louis XIV, Mémoires, S. 81.

79 Vgl. Colbert, Lettres, Bd. II, S. 35. 
mal war es Colbert, der rund sechs Wochen später zu der Verschärfung der für Fouquet vorgesehenen Strafe beitrug. Um seinen Gegner zu vernichten, wollte er dem König beweisen, dass der Finanzminister nicht nur königliche Gelder veruntreute, sondern zudem eine Gefahr für die königliche Macht darstellte. Ein von ihm entsandter Spion berichtete Mitte Juni, dass Fouquet unter dem Deckmantel von Handelstätigkeiten seine 1658 gekaufte Insel BelleÎle zu einer Festung ausbauen ließ. Neben den rund tausendfünfhundert Arbeitern, die auf der Insel beschäftigt waren, befanden sich dort ausreichend Kanonen, Waffen und Munition für die Versorgung einer sechstausend Mann starken Armee. Der Bericht erzielte die gewünschte Wirkung. Ludwig XIV. beschloss, Fouquet zu verhaften: »Je ne m'étais d'abord proposé de l'éloigner des affaires; mais ayant depuis considéré que de l'humeur inquiète dont il était, il ne supporterait point ce changement de fortune sans tenter quelque chose de nouveau, je pensai qu'il était plus sûr de l'arrêter « ${ }^{80}$. Wegen der bevorstehenden Erntezeit und der damit verbundenen Steuereintreibungen entschied man, mit der Verhaftung bis Ende August zu warten und sie gründlich vorzubereiten $^{81}$. Bei den Vorbereitungen des Sturzes stellten sich zwei Probleme: Zum einen genoss Fouquet als procureur général, der allein der Gerichtsbarkeit des Parlaments unterworfen war, in dem er zahlreiche Anhänger hatte, nahezu Immunität, zum anderen hatte er in der Person Annas von Österreich, deren Einfluss der König noch immer unterlag, eine langjährige Beschützerin, die überdies hohe Pensionszahlungen von Fouquet erhielt. Vor der eigentlichen Verhaftung galt es, diese Hindernisse zu überwinden. Colbert legte dem König nahe, dass zur Verurteilung des Finanzministers ein königliches Sondergericht, eine chambre de justice, eingerichtet werden müsse, um Einfluss auf die Zusammenstellung der Kommission nehmen zu können. Um den Finanzminister der Zuständigkeit dieses Gerichtes zu unterwerfen, musste man ihn dazu bringen, sein Amt als procureur général zu verkaufen. Hierfür erdachte man sich folgende List: Der König ließ vor Fouquet den Wunsch verlauten, persönlich über eine Summe von rund einer Million Livres zu verfügen und bat seinen surintendant, über mögliche Wege zur Beschaffung dieser Summe nachzudenken. Zugleich legte er Fouquet die Notwendigkeit dar, das Parlament zu reformieren, und gab ihm zu verstehen, dass Fouquets Fähigkeiten nach einer Restrukturierung dort nicht mehr adäquat zum Einsatz kommen würden. Ludwig stellte dem ehrgeizigen Minister sowohl das Amt des Kanzlers nach dem Tod Séguiers als auch die Aufnahme in den ordre $d u$ Saint-Esprit in Aussicht. Damit hatte er Fouquet auf die gewünschte Fährte gelockt. Der Finanzminister bot dem König schon wenige Tage später den

${ }^{80}$ LouIs XIV, Mémoires, S.81f.

81 „L'été, où nous étions alors, était celle des saisons de l'année où ces innovations se faisaient avec le plus de désavantage, outre que je voulais avant toutes choses avoir un fonds en main de quatre millions, pour les besoins qui pourraient survenir. Ainsi je me résolus d'attendre l'automne pour exécuter ce projet« (ibid., S.82). 
Verkauf des genannten Amtes an, um seinem Wunsch zu entsprechen ${ }^{82}$. Der König erklärte sich mit dem Opfer einverstanden, und Fouquet machte sich nun daran, den Verkauf des Amtes in die Wege zu leiten ${ }^{83}$. Das Einverständnis der Königinmutter zum bevorstehenden Sturz Fouquets versuchte man mittels einer von Colbert erdachten List zu erhalten: Mit der Unterstützung der Duchesse de Chevreuse, einer alten Freundin Annas von Österreich, die mit einem Gegner Fouquets verheiratet war, konnte man die Königinmutter von der Notwendigkeit seines Sturzes überzeugen.

Ende Juni entwarf Ludwig einen ersten Plan für die Verhaftung. Es galt, einen Ort zu finden, der dem Finanzminister wenig Schutz durch Verbündete gewährte und zugleich kein Misstrauen auf seiner Seite erweckte. Die Wahl des Königs fiel auf die Bretagne, aus der ein Zweig der Familie Fouquets stammte. Es handelte sich um eine dem König unbekannte Provinz - ein Umstand, der ihm als Vorwand diente, für Ende August in Nantes die états provinciaux unter seinem Vorsitz einzuberufen ${ }^{84}$. Dem Abbé Choisy zufolge drohte dieser Plan im Juli verworfen zu werden: Nachdem der surintendant am 12. dieses Monats in Vaux den Bruder des Königs und seine Gemahlin empfangen hatte, erbat auch der König einen Empfang in Vaux. Dem Abbé nach fasste Ludwig den Plan, den surintendant bereits auf dem Fest zu verhaften $^{85}$. Die Königinmutter brachte den König von dieser Idee ab:

Le roi [...] l'engagea à lui donner une fête dans sa belle maison de Vaux, résolu de le faire arrêter au milieu des hautbois et des violons, dans un lieu qui se pouvait dire une preuve parlante de la dissipation des finances. Mais avant l'exécution, n'ayant pu s'empêcher d'en faire la confidence à la reine mère, elle lui dit tant de raisons pour l'en empêcher, qu'il résolut dès lors de faire le voyage de Nantes ${ }^{86}$.

Fouquet blieben die Vorbereitungen zu seinem Sturz nicht gänzlich verborgen. Er verfügte über ein gut funktionierendes Netzwerk von Zuträgern und war über das Komplott der Duchesse de Chevreuse sowie die Schritte Col-

${ }^{82}$ Fouquet erklärte sich sogar bereit, Vaux und Belle-Île zu verkaufen, um dem König die gewünschten Geldmittel zur Verfügung stellen zu können - ein Zeichen seiner uneingeschränkten Königstreue (Fouquet, Euvres, Bd. VII, S.366; siehe auch: Choisy, Mémoires (2005), S.143: »Je vendrais de bon cœur tout ce que j'ai au monde pour donner de l'argent au Roi $\ll)$.

83 Vor dem Verkauf des Amtes musste noch ein Hindernis überwunden werden: Basile, der die Anwartschaft auf das Amt besaß, erklärte sich erst gegen die Zahlung einer Summe von 400000 Livres mit dem Verkauf einverstanden, so dass sich die Kaufsumme entsprechend erhöhte.

${ }^{84}$ Vgl. Dessert, Fouquet, S. $240 f$.

85 Einige sahen sogar das Fest selbst als Grund für die königliche Ungnade an (zum Beispiel Montglat, Mémoires, S.352, zitiert nach: Niderst, Siècle de Louis XIV, S. 675), eine Meinung, die zunächst von vielen Historikern übernommen wurde.

${ }^{86}$ Cholsy, Mémoires (2005), S.144. Brienne zufolge wies die Königinmutter Ludwig darauf hin, dass eine Verhaftung in Vaux dem König keinen Ruhm bringen würde: »Ah! mon fils, cette action ne vous fera guère d'honneur: ce pauvre homme se ruine pour vous faire bonne chère et vous le ferez arrêter prisonnier dans sa maison!« (BRIENNE, Mémoires, Bd. III, S. 49). 
berts unterrichtet. Seine Informantin Mme d'Huxelles warnte ihn im August vor dem freundlichen Ton, mit dem Ludwig bis zuletzt seinen Finanzminister empfing, sowie vor der geplanten Reise in die Bretagne:

Vous devez même vous défier du bon accueil et du bon visage que vous fait le roi [...]. L'on m'a promis de m'apprendre des choses qui sont de la dernière conséquence sur cela, sur le voyage de Bretagne, sur certaines résolutions très secrètes du roi et sur des mesures prises contre vous ${ }^{87}$.

Trotz aller Warnungen zeigte sich Fouquet sorglos und war überzeugt von der Gunst des Königs, der ihn höflich und zuvorkommend behandelte. Dieser jedoch ärgerte sich über Fouquets Verhalten, dessen Fehler im Umgang mit dem Monarchen sich häuften: Er verlangte zusätzliche Audienzen bei Ludwig XIV. und versuchte weiterhin, Klienten in der Umgebung des Königs zu gewinnen. Sein Fehlverhalten erreichte einen Höhepunkt, als er sich Mlle de La Vallière, der neuen Maitresse Ludwigs, näherte, um sie für seine Interessen zu gewinnen. Diese wies sein Angebot empört zurück und denunzierte ihn umgehend bei ihrem Liebhaber ${ }^{88}$.

Am 10. August stand einer Verhaftung des Finanzministers nichts mehr im Wege. Fouquet verkaufte an diesem Tag das Amt des procureur général an Achille de Harlay. Doch vor seinem Sturz sollte er noch einmal die Chance bekommen, seinen König gnädig zu stimmen. Trotz der geplanten Verhaftung in Nantes beschloss Ludwig, an dem Fest in Vaux teilzunehmen. Der Prunk, den der Finanzminister zur Schau stellte, bestärkte den König in seinem Entschluss, und noch während des Festes kursierten Gerüchte über eine Festnahme des Gastgebers ${ }^{89}$. Der Zorn des Monarchen war nicht mehr zu bremsen: »Piqué de la magnificence de Vaux, qui effaçait de bien loin Fontainebleau et toutes les autres maisons royales, [le roi] n'avait pas pu s'empêcher de dire à la reine mère: >Ah! Madame, est-ce que nous ne ferons pas rendre gorge à tous ces gens-là? « ${ }^{90}$. In dem ihm zugeschriebenen »Journal secret « ließ der König seiner Empörung freien Lauf:

Le 17 d'août. - Ce jour, nous fûmes priés à une fête offerte en son château de Vaux-le-Vicomte par le Surintendant: Souhaitant de nous éblouir par son luxe et ses richesses (mal acquises), M. Fouquet acheva ainsi de se perdre en mon esprit. Comment un ministre peut-il en toute innocence mener ce train royal? De quels abymes d'iniquité tire-t-il ses ressources? Comment peut-il entretenir les meilleurs architectes, peintres et jardiniers, sinon en puisant dans les caisses de l'État? ${ }^{91}$

87 Zitiert nach: Petitfils, Fouquet, S. 354.

88 Cholsy, Mémoires (2005), S. 141.

89 »Au milieu de la fête, M. le surintendant reçut un billet de Mme du Plessis-Bellière, qui lui donnait avis qu'on devait l'arrêter à Vaux: mais la reine mère avait fait changer l'ordre« (ibid., S. 146).

${ }^{90}$ Ibid.

91 Louis XIV, Journal secret, S.23. Der Vorwurf des Königs, Fouquet hätte seinen Schlossbau aus der Staatskasse finanziert, ist unberechtigt. Seit 1653 fungierte Fouquet hingegen selbst als Kreditgeber der Krone und lieh dem Staat zwischen 1653 und 1661 rund 30 Millionen Livres (Kettering, Patrons, S.193). Der Vergleich seiner Aktiva und Passiva im 
Noch immer wollte Fouquet die ihm drohende Gefahr nicht wahrhaben. Einige Tage nach dem Fest befragte er seinen engen Freund Gourville, wie man bei Hofe über ihn denke: »Je lui répondis que les uns disaient qu'il allait être déclaré premier ministre, et les autres qu'il y avait une grande cabale contre lui pour le perdre ${ }^{92}$.

Fouquet war erschöpft: Seit dem 26. Juli litt er unter Sumpffieber mit regelmäßigen Fieberattacken, die jedes Mal ein Delirium verursachten. Am 28. August machte sich der kranke Finanzminister auf den Weg nach Nantes. Sein Verhalten an den Tagen vor der Verhaftung zeugt von einem verwirrten Geisteszustand. Auf der einen Seite bezeugte er immer wieder, dass er nicht an eine Gefahr für seine Person glaube, dass Ludwig vielmehr Vorbereitungen träfe, Colbert zu verhaften ${ }^{93}$, auf der anderen Seite bezog er in Nantes ein Haus, welches über einen direkten Fluchtweg zum Fluss verfügte und erklärte Brienne zugleich, dass er eine Flucht für zwecklos halte, da ihm an keinem Hof Europas Schutz gewährt werden würde ${ }^{94}$. Am 5.September, dem dreiundzwanzigsten Geburtstag des Königs, wurde Fouquet im Anschluss an eine Sitzung des Ministerrates von d'Artagnan, capitaine lieutenant der Musketiere, verhaftet. Während Ludwig den Höflingen erklärte, dass er von nun an keinen surintendant des Finances mehr benötige, wurde der gestürzte Minister in einem vergitterten Wagen nach Angers gebracht. Am Ende seiner kurzen Karriere fand er sich in der Stadt wieder, von der der Aufstieg seiner Familie ihren Ausgang genommen hatte.

Nicolas Fouquet profitierte wie kein anderer von den Möglichkeiten eines gesellschaftlichen Aufstiegs in seiner Epoche, um am Ende wie kein zweiter an ihnen zu scheitern. Sein Aufstieg gelang ihm dank der zielstrebigen Bemühungen seines Vaters und seiner engen Bindungen an die Premierminister Richelieu und Mazarin sowie seiner steten Loyalität dem Königshaus gegenüber. In seiner Position als Finanzminister war er extremen Anfeindungen ausgesetzt, die ihm schließlich in der Person Colberts zum Verhängnis wurden. Der Finanzminister, der auch in Zeiten chronischen Geldmangels ohne Unterlass Kapital für die Krone aufgetrieben hatte, wurde 1661 für die miserable Finanzlage verantwortlich gemacht ${ }^{95}$. Er büßte zugleich für die Maß-

Jahr seiner Verhaftung zeigt, dass von einer Bereicherung nicht die Rede sein konnte: 15442473 Livres (Aktiva) standen 15531725 Livres (Passiva) gegenüber (DEssert, Fouquet, S.348-352).

92 Gourville, Mémoires, S. 133.

93 »C'est Colbert qui sera arrêté et non moi [...]. C'est moi [...] qui ai donné au roi tous ces avis afin de mieux couvrir notre jeu« (BRIENne, zitiert nach: Niderst, Siècle de Louis XIV, S.697).

94 »Mais m'enfuirai-je? C'est ce qu'on serait peut-être bien aise que je fisse. Me cacheraije? Cela serait peu expédient; car quel prince [...] oserait me donner sa protection? « (ibid., S. 693).

95 Inwieweit Fouquet, der während seiner Amtszeit die Korruption im Finanzwesen nicht zu mindern wusste, selbst Anteil an der miserablen Finanzlage hatte, kann im Rahmen 
losigkeit des verstorbenen Premierministers Mazarin, der Ludwig XIV. mit Colbert seinen engsten Mitarbeiter und Vertreter seiner eigenen Interessen auch über seinen Tod hinaus an die Seite gestellt hatte ${ }^{96}$. Der Fall Fouquets wurde zu einem Symbol der von Ludwig erlangten Selbständigkeit nach dem Tode des Premierministers. Mit seinem Sturz musste der bürgerliche Parvenu für die Malaisen seiner Zeit büßen.

Das Leben Nicolas Fouquets war geprägt von dem glühenden Verlangen nach gesellschaftlichem wie politischem Aufstieg. Sein Ehrgeiz brachte ihm einen raren Ministerposten. Die Übernahme des Amtes als Finanzminister war zugleich die Geburtsstunde des Kulturpatrons Nicolas Fouquet. Minister und Mäzen standen in einem steten Wechselspiel zueinander. Die politische Entwicklung bedingte die Handlungen des Kunstförderers Fouquet. Bereits in seinem Elternhaus und bei den Jesuiten hatte er die Vorzüge der Kulturpatronage für die gesellschaftliche wie berufliche Etablierung schätzen gelernt. Die Förderung der Künste bot eine hervorragende Möglichkeit der sozialen Distinktion, die für einen Aufstieg unerlässlich war. Herrschaftliche Bauten, Aufsehen erregende Sammlungen, literarische Lobreden und festliche Empfänge waren ein geeignetes Mittel, sich gesellschaftliches Ansehen zu verschaffen und damit die eigene Stellung und politische Macht zu legitimieren.

Um zunächst seine Karriere voranzutreiben und am Ende seinem Sturz entgegenzuwirken, tat sich der Finanzminister als Kunstförderer hervor. Die erste Phase seiner Kulturpatronage (1653-1655) war - mit dem Kauf einer repräsentativen Schlossanlage, dem Aufbau verschiedener Kollektionen und der Unterstützung von Literaten - seiner Etablierung in den Reihen der Minister gewidmet. Mit seinem politischen Schicksal jedoch änderte sich auch die Art seines Mäzenatentums. In der zweiten Phase seiner Kulturpatronage (1656-1661) machte der Finanzminister die Künste für seine eigenen Interessen dienstbar und versuchte auf diese Weise, die Gunst des Königs zu erlangen, um seine gefährdete Stellung zu stabilisieren und zugleich den Weg für einen weiteren Aufstieg zu bereiten.

In dem Maße, in welchem sich die Zielsetzung der beiden Phasen unterscheidet, divergiert auch die Art der Kulturförderung und die durch die Förderung Fouquets entstandenen Kunstwerke. Die Kulturpatronage des Finanzministers ist damit eine direkte Antwort auf die jeweils aktuelle politische Lage, in der sich Nicolas Fouquet zwischen 1653 und 1661 befand.

dieser Arbeit nicht untersucht werden. Es ist jedoch anzunehmen, dass auch er - bewusst oder unbewusst - einen Teil der Schuld zu tragen hatte.

96 Dessert, Louis XIV, legt ausführlich dar, welche Machtstrukturen im Jahr 1661 zum Sturz des Finanzministers führten. Er erklärt, auf welche Weise Nicolas Fouquet Büßer für die durch die bis 1659 stetig anhaltenden Kriege und die gestiegenen Hofhaltungskosten hervorgerufene Finanzkrise und Katalysator für die maßlose Bereicherung Mazarins an der Staatskasse wurde. Dieser hatte sein Privatvermögen, bei einem durchschnittlichen Jahreseinkommen von 2 Millionen Livres, zwischen 1653 und 1661 auf rund 40 Millionen ansteigen lassen und somit die Finanzkrise weiter vorangetrieben. 